Journal of Engineering and Applied Sciences 14 (Special Issue 6): 9467-9476, 2019

ISSN: 1816-949X

(C) Medwell Journals, 2019

\title{
Layout Optimization of Sewer Networks by Adaptive Genetic Algorithmin A Hybrid Model
}

\author{
Waqed H. Hassan and Zahraa H. Attiyah \\ University of Kerbala, Kerbala, Iraq
}

\begin{abstract}
The problem of optimal layout is one of the most difficult problems. This is due to the large size of the search space, which expands rapidly as the size of the network increases. This paper introduces an adaptive procedure to improve the efficiency of genetic algorithm (GA) formulation in GA-TGA optimization model of the optimum layout design of sewer networks. Adaptive strategy helps the designer develop adaptive genetic algorithms in which method operators are systematically adapted to the constraints of the layout problem. The adaptive selection operator keeps the genetic algorithm in feasible region of the search space and consequently improve the performance of optimization in terms of speed. The formulation of selection and crossover lead to not need to discard or repair unfeasible solutions or apply penalty factors to the cost function as commonly used in the principles of genetic algorithms. Four different selection methods will be used with the GA. In MATLAB code, the optimization model implemented. Benchmark example and case study of sewer networks are used to test the present method. This method has proven to be effective in terms of solution optimality and the resulting convergence characteristics. Additionally, the method proves itself capable of finding an optimal, or near optimal solution.
\end{abstract}

Key words: Sewer networks, genetic algorithms, optimization, case study, fitness function

\section{INTRODUCTION}

Sewer networks are the basic infrastructure of urban cities and have a direct influence on public health. The task of building a new sewer system is a very expensive and difficult task especially in densely populated cities. Therefore, the issue has prompted engineers and researchers to develop optimization methods to obtain the most effective and efficient designs in terms of cost.

To find optimum sewer layout "system layout" is surely not an easy work for engineers. The difficulty is mainly due to two important factors: the complexity of the system's environment and the huge number of possible design alternatives. Particularly in the large networks necessary for urban sewer system, manual calculation limits the evaluation of alternatives. This has inspired many computerized optimization model studies. Some researchers, have addressed the problem of layout geometry determination of networks using different optimization methods (Mays et al., 1976; Rowell and Barnes, 1982; Morgan and Goulter, 1985; Walski, 1985; Walters, 1992; Walters and Lohbeck, 1993; Davidson and Goulter, 1995; Walters and Smith, 1995; Davidson, 1999; Geem et al., 2000; Hassan et al., 2018).

Genetic algorithms are best suited to solving combinatorial optimization problems with very large solution spaces which cannot be solved using more conventional optimization methods. The GAs were originally conceived by Holland (1975) and have since been further developed by De Jong (1975),
Goldberg (1989) and subsequently by many others (Miettinen et al., 1999). The use of genetic algorithms for effective and efficient research of the optimal layout solution for sewer network is governed by several factors such as coding type, fitness function, size of population, three main operators (choice, mating and mutation), penalty method, number of generation and finally, the most important is the size of the search space. This paper proposed an adaptive procedure to improve the efficiency of genetic algorithm formulation to reduce the search space. A model, GA-TGA, based on the proposed methodology is developed and first tested and verified for its efficiency and effectiveness on two previously benchmark problems networks (Hassan and Atiyah, 2019). Here, it is applied to the optimal layout design of case study in Karbala city, Iraq. The results obtained indicate that the modified GA with reduction in search space proposed herein is effective, especially for large practical networks.

\section{ADAPTIVE GENETIC ALGORITHM FORMULATION}

The genetic algorithm dates back to the late 1970's in Holland (1975) work such as evolutionary models. Its popularity has grown considerably since then and there are many engineering applications now. The algorithm uses crossover operators and mutation to move from one generation to the next to create solutions and the selection operator works to select individuals from generation to 
next. Genetic algorithms belong to a category of powerful research techniques that rely on genetic inheritance and natural selection in Darwinian metaphor. These algorithms maintain a finite memory of individual points on the search landscape known as the "population". Typically, members of the population are represented as a string, each of which has a standard value associated with it that reflects its quality or fitness. The research may seem to be a iterative application to a number of operators such as selection, crossover and mutation, the goal is to produce progressive individuals gradually. One of the larger drawbacks of genetic algorithms is that they require a large number of function evaluations to achieve convergence. Each functional evaluation requires a complete simulation of the system for a long time, a process that is highly cost-effective. This concludes that GA is a time-consuming optimization system. It can take an improved runtime for a large distribution system to take up to a few days on a modern personal computer. Various reasons can be stated why long running times may be problematic.

In the present study, an attempt is made to improve GA for the design layout of practical networks, by increasing its efficiency and effectiveness. In the adaptive GA, a integer representation, instead of using only the basic selection method, four different selection methods are used, simple objective function, order crossover method and a one gene mutation operator. A methodology based on critical path method is suggested herein to reduce the search space. The GA-TGA model is developed using MATLAB code.

The improved GA formulation was achieved by using different selection methods providing an improvement compared with the simple GA. As follows, a brief description of the four selection methods.

Roulette Wheel Selection (RWS): The roulette wheel is the simplest and traditional random selection method proposed by the Holland (1975). It is classified under a proportional selection as individuals select on the basis of a probability proportionate with their fitness. Assigned for each individual a part on the roulette wheel. The size of each part in the roulette wheel is proportional to the value of individual's fitness, The higher the value, the greater the part (Hassan, 2019):

$$
p_{i}=\frac{f_{i}}{\sum_{j=1}^{n} f_{j}}
$$

- $\quad$ Stochastic Universal Sampling (SUS): Stochastic Universal Sampling is introduced by Baker (1987), is quite similar to Roulette wheel selection. However, instead of spinning the roulette wheel $\mathrm{n}$ times as described in Roulette Wheel Selection, in this technique one can spin the Roulette Wheel just once, but after determining $n$ points in the Wheel, where $n$ is a population size. Then choose $\mathrm{n}$ chromosomes that situated in front of the determined points

- Exponential Rank Selection (ERS): This is based on the chromosomes' rank instead of their fitness. The rank of 1 is granted for the worst chromosome, while the best chromosome is given the rank of $n$. Thus, based on its rank, each chromosome (i) has the probability of being selected given the expression (Jebari and Madiafi, 2013):

$$
\mathrm{p}(\mathrm{i})=1.0 \times \mathrm{e}^{\left(\frac{-\operatorname{rank}(\mathrm{i})}{\mathrm{c}}\right)}
$$

Where:

$$
c=\frac{(2 n \times(n-1))}{(6(n-1)+n)}
$$

- $\quad$ Random Selection (RMS): In this method parents are randomly selected from the current population. There is no strategy for selection pressure to certain individuals and therefore prefer to avoid this method usually

- GA-TGA model: The GAs are often used in combination with a problem-specific or local search procedure, especially in commercial applications (Goldberg and Voessner, 1999). The goal of using a problem specific search method is to improve the efficiency of the GA, either in terms of the time required to find a good solution or the quality of the solution found. Early hybrid GAs were introduced by Smith (1985) and Grefenstette et al. (1985) are commonly used today in serious GA applications (Goldberg and Voessner, 1999)

The GA-TGA a technique combined genetic algorithm with tree growing algorithm, which uses a suitable growth algorithm TGA to avoid the problems associated with configuring of infeasible solutions, the following is a brief description of the model:

- Coded the design variable: This coded string is similar to the structure of a chromosome of genetic code. A selected mapping between the coded sub-strings and the design variables associates the artificial genetic code with a pipe network design

- Generation of initial population by using TGA: The trees produced will on average be biased towards those which diverge from a root node speci ed on the base graph. This is a useful feature of the algorithm as it closely mimics the characteristics of the natural plant growth and of most engineering tree networks involving flows 
- Fitness function: For each layout in the population will be find the fitness, which is represented the inverse of the objective function of each layout. Walters and Smith (1995) suggested a simplified cost function to found the cost of layout based on the length and concave function of the flow rate per pipe, as shown follows:

Fitness function $=1$ objective function

$$
\text { Total cost }=\sum_{\mathrm{i}=1}^{\mathrm{m}} \mathrm{l}_{\mathrm{i}} \sqrt{\mathrm{Q}_{\mathrm{i}}}
$$

where, total cost is the layout objective function, $\mathrm{L}$ is sewer length and $\mathrm{Q}$ is accumulated sewer flow rate that is indirectly obtained with the layout configuration

- Generation of a new population using the selection, crossover and mutation operators. They occur with some specified probability

- Production of successive generations: A new generation is produced when using the above three operators in steps 3 and 4 . The GA repeats the process to generate successive generations. The least cost strings are stored and updated as cheaper cost alternatives are generated

\section{APPLICATIONS}

The applicability and ef ciency of the formulations described in the previous section are tested in this section against benchmark example in the literature and the real case study. The rst example to be considered is that of a simple network shown in Fig. 1 proposed and solved by Walters and Smith (1995) using evolutionary programing. More details about this example are found in the cited reference.

Figure 2 shows the typical convergence curve for a number of generations during the evolutionary process to reach the optimal solution cost by GA-TGA method with the RWS, ERS,SUS and RMS selection methods respectively, for directed base graph of the benchmark example.

The results were obtained with an order crossover method $(\mathrm{Pc}=0.9)$ and a one gene mutation per chromosome with probability of mutation $\mathrm{Pm}=0.5$. As expected, the number of generations required to reach the final solution is improved by using different methods of selection. The optimum objective function of solution 5218 units was obtained with the Exponential Rank Selection (ERS) method, within 306 generations this is the best and fastest method of selection. As for the RWS

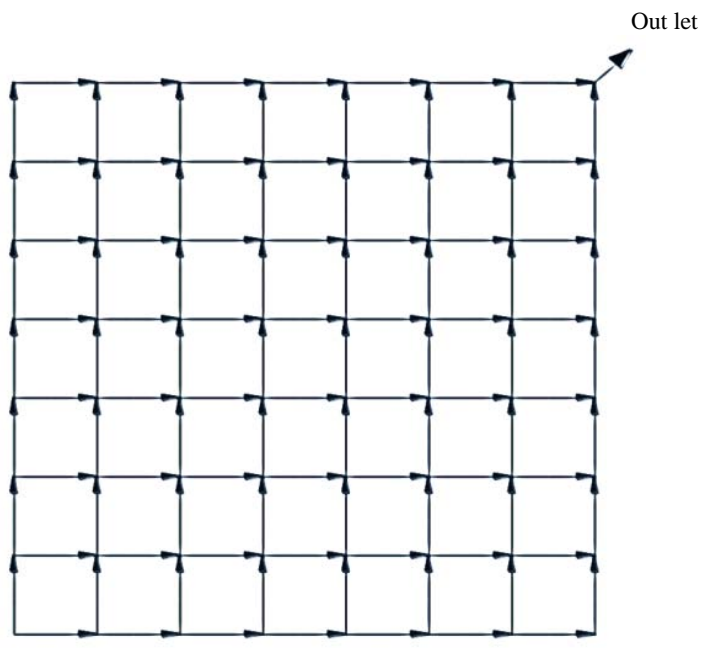

Fig. 1: Directed base graph Walters and Smith (1995)

method, here their little disparity in costs because total cost for each individual in population are close, so the chance is equal.RMS selection method the result of thismethod show that the data are irregular so this method do not work with the proposed model.

Case study: A first sector from Al-Amil quarter in karbala city, Iraq was used exam the new proposed method. Karbala is located in the central region of Iraq. The city of Karbala was chosen for several reasons. There is no significant change in terrain heights and therefore, the designer cannot see the clear tracking of natural land slopes to the specific outlet. In such areas, there is often a lot of possible options for connecting sewers networks and the location of the network outlet. There is only one outlet discharging to sewage treatment plant. The design discharge is $250 \mathrm{~L} / \mathrm{ca} /$ day. This value is provided by the sewer network which includes 216 nodes and 215 pipes, the total length of network $(8.227 \mathrm{~km})$. It forms about (0.485) and the layout of the present network shown in Fig. 3. When using the objective function to calculate the total cost of actual design for layout of network as build, a total cost was obtained equal 450.92 units. Table 1 presents the data characteristics as build for this network and information of actual layout design by manually.

Figure 4 shows the performance of the proposed GA-TGA model with the case study. The result was obtained with an Exponential Rank Selection (ERS) method was the best selection method worked with present model in previous benchmark example, Order crossover $(\mathrm{OX})$, the probability of crossover $\mathrm{Pc}=0.9$, one-gene mutation per chromosome, the probability of mutation $\mathrm{Pm}=0.5$ and population size equal to 
J. Eng. Applied Sci., 14 (Special Issue 6): 9467-9476, 2019
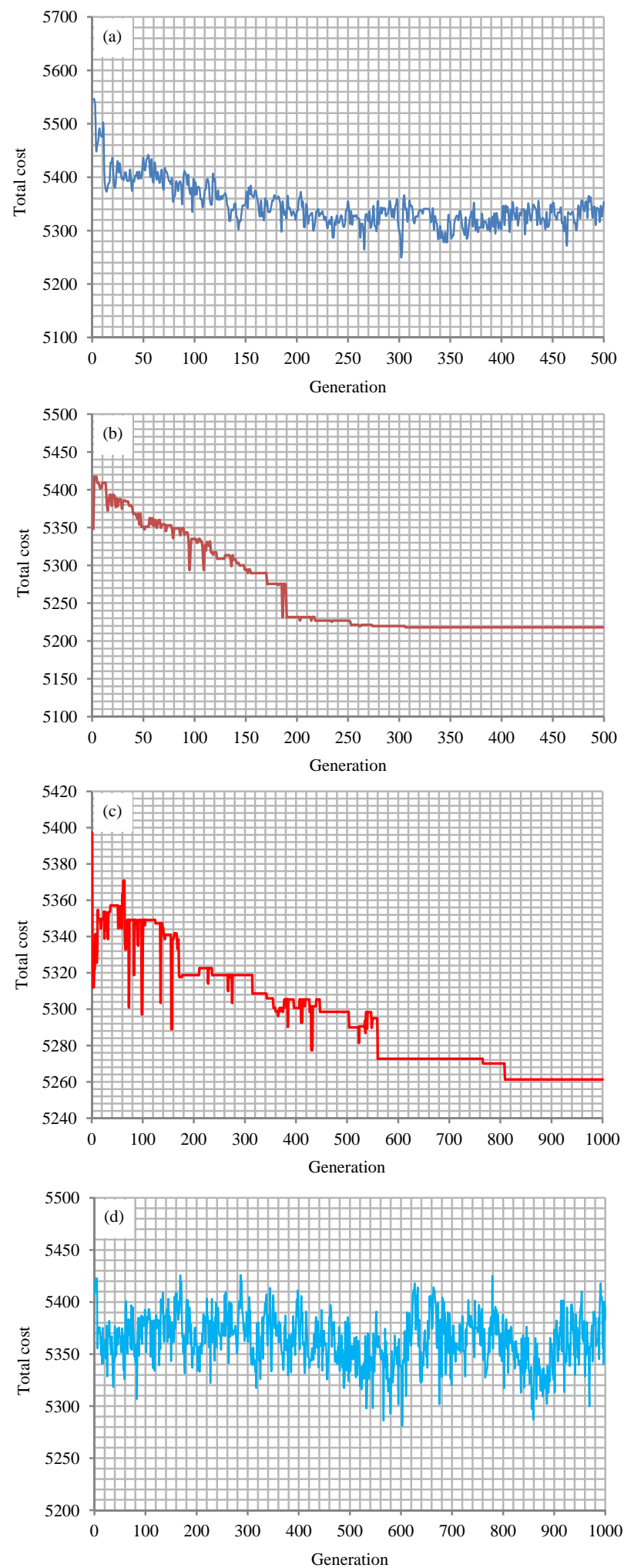

Fig. 2(a-d): Convergence characteristics of generations for different selection methods (a) RWS, (b) ERS, (c) SUS and (d) RMS, by the present model 


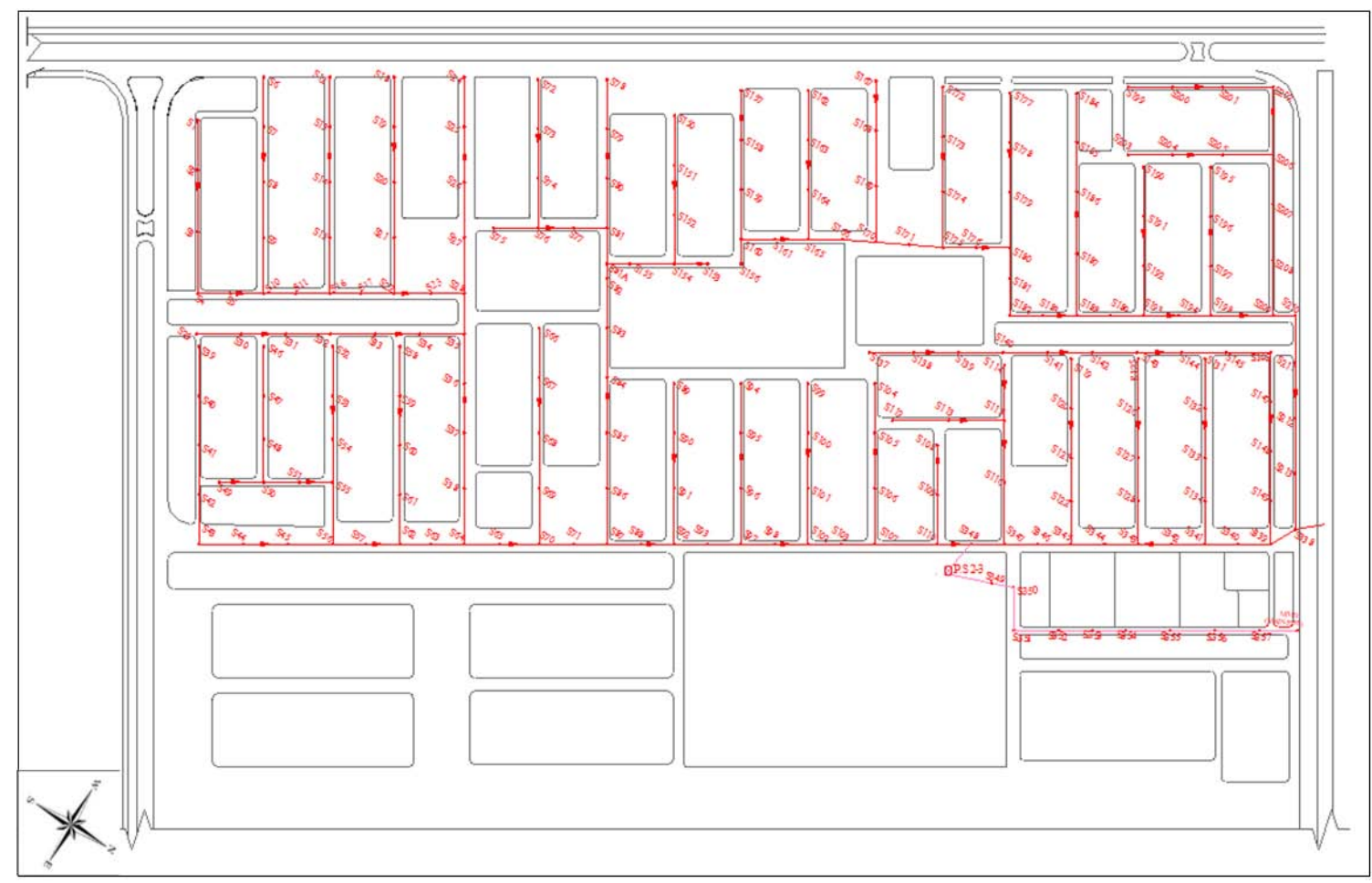

Fig. 3: Existing layout of case study (first sector from Al-Amil district)

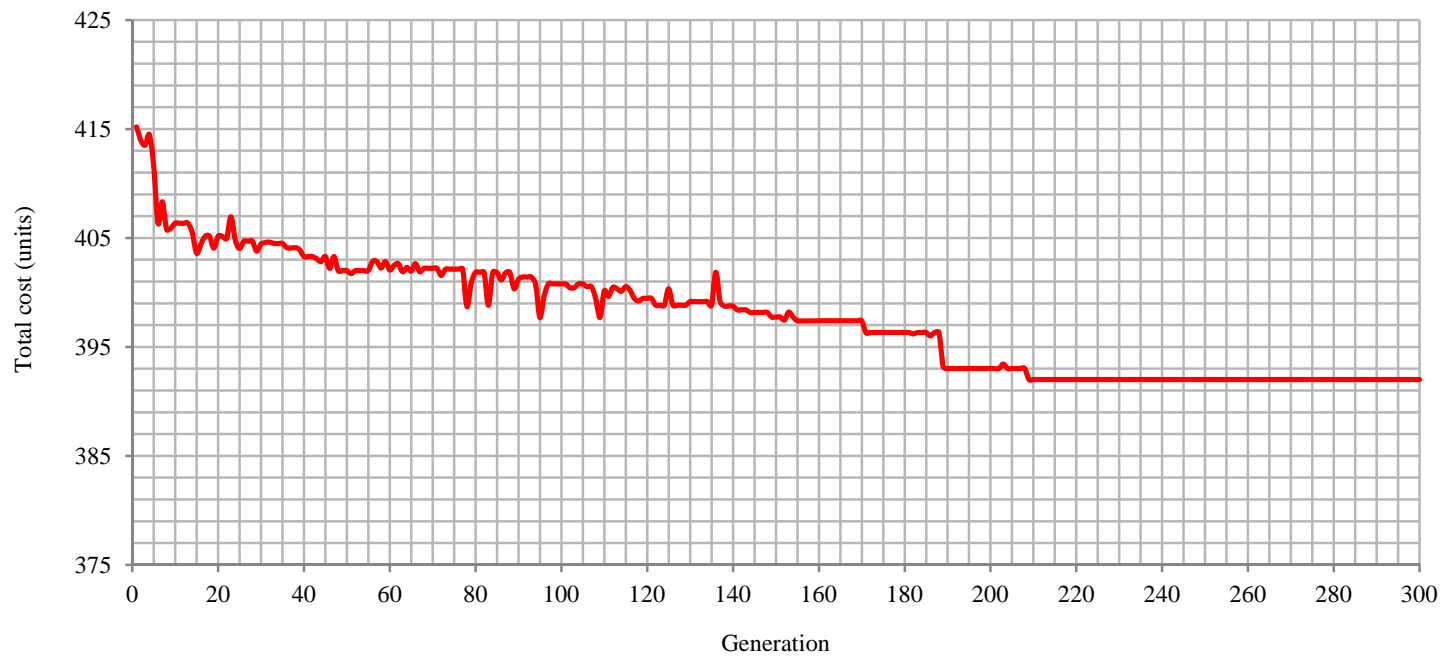

Fig. 4: Optimum cost solution by the proposed method for the case study

200 chromosomes. Repeatedly running the program, the optimum layout with minimum cost equal 392.0 units is gained in 209 generations as shown in Fig. 5, resulting in a reduction of about $13.05 \%$, this reduction is relatively very good because of the initial cost of establishing sewerage networks, which are an important part of the city's infrastructure. Table 2 presents the data characteristics for optimum layout design of the case study by proposed model. 


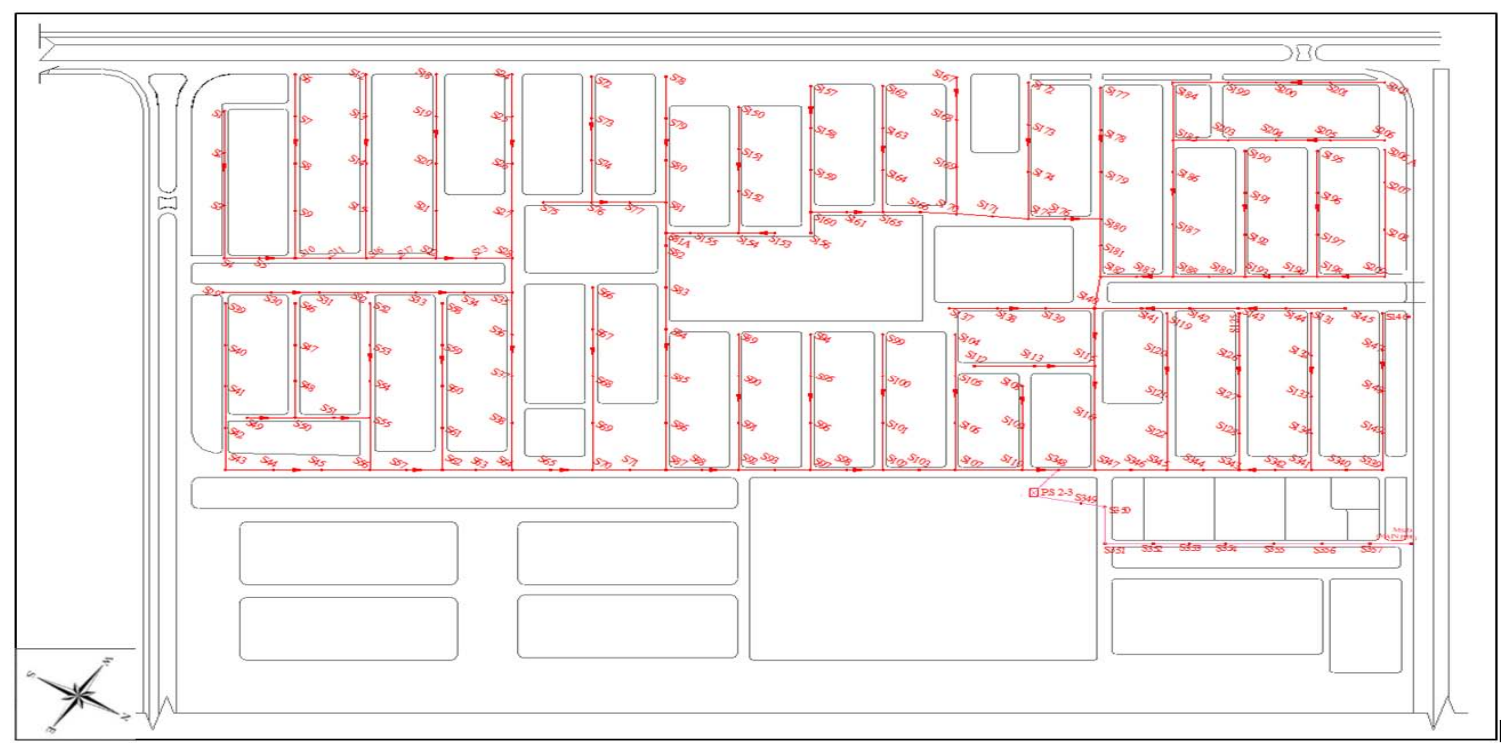

Fig. 5: Optimum layout of case study obtained by proposed model

Table 1: Existing layout design for the first case study

\begin{tabular}{|c|c|c|c|c|c|c|c|}
\hline \multicolumn{2}{|c|}{ Link from to } & \multirow{2}{*}{$\frac{\text { Length (m) }}{40}$} & \multirow{2}{*}{$\frac{\mathrm{Q} \text { design }\left(\mathrm{m}^{3} \mathrm{sec}^{-1}\right)}{0.000355}$} & \multicolumn{2}{|c|}{ Link from to } & \multirow{2}{*}{$\frac{\text { Length }(\mathrm{m})}{30}$} & \multirow{2}{*}{$\frac{\mathrm{Q} \text { design }\left(\mathrm{m}^{3} \mathrm{sec}^{-1}\right)}{0.014660}$} \\
\hline 1 & 2 & & & 103 & 107 & & \\
\hline 2 & 3 & 50 & 0.000790 & 104 & 105 & 40 & 0.000355 \\
\hline 3 & 4 & 50 & 0.001224 & 105 & 106 & 45 & 0.000746 \\
\hline 4 & 5 & 29 & 0.001474 & 106 & 107 & 45 & 0.001137 \\
\hline 5 & 10 & 30 & 0.001735 & 107 & 110 & 56 & 0.016276 \\
\hline 6 & 7 & 40 & 0.000355 & 108 & 109 & 40 & 0.000355 \\
\hline 7 & 8 & 45 & 0.000746 & 109 & 110 & 40 & 0.000703 \\
\hline 8 & 9 & 45 & 0.001137 & 110 & 348 & 30 & 0.017230 \\
\hline 9 & 10 & 45 & 0.001527 & 112 & 113 & 50 & 0.000443 \\
\hline 10 & 11 & 29 & 0.003506 & 113 & 115 & 50 & 0.000877 \\
\hline 11 & 16 & 30 & 0.003766 & 114 & 115 & 45 & 0.000399 \\
\hline 12 & 13 & 40 & 0.000355 & 115 & 116 & 50 & 0.001701 \\
\hline 13 & 14 & 45 & 0.000746 & 116 & 347 & 50 & 0.002135 \\
\hline 14 & 15 & 45 & 0.001137 & 119 & 120 & 40 & 0.000355 \\
\hline 15 & 16 & 45 & 0.001527 & 120 & 121 & 40 & 0.000703 \\
\hline 16 & 17 & 28 & 0.005528 & 121 & 122 & 35 & 0.001006 \\
\hline 17 & 22 & 30 & 0.005789 & 122 & 345 & 35 & 0.001310 \\
\hline 18 & 19 & 40 & 0.000355 & 125 & 126 & 40 & 0.000355 \\
\hline 19 & 20 & 45 & 0.000746 & 126 & 127 & 40 & 0.000703 \\
\hline 20 & 21 & 45 & 0.001137 & 127 & 128 & 35 & 0.001006 \\
\hline 21 & 22 & 45 & 0.001527 & 128 & 343 & 35 & 0.001310 \\
\hline 22 & 23 & 33 & 0.007595 & 131 & 132 & 40 & 0.000355 \\
\hline 23 & 28 & 30 & 0.007855 & 132 & 133 & 40 & 0.000703 \\
\hline 24 & 25 & 40 & 0.000355 & 133 & 134 & 35 & 0.001006 \\
\hline 25 & 26 & 45 & 0.000746 & 134 & 341 & 35 & 0.001310 \\
\hline 26 & 27 & 45 & 0.001137 & 137 & 138 & 40 & 0.000355 \\
\hline 27 & 28 & 45 & 0.001527 & 138 & 139 & 40 & 0.002873 \\
\hline 28 & 35 & 33 & 0.009660 & 139 & 140 & 40 & 0.003220 \\
\hline 29 & 30 & 40 & 0.000355 & 140 & 141 & 40 & 0.003567 \\
\hline 30 & 31 & 40 & 0.000703 & 141 & 142 & 40 & 0.003914 \\
\hline 31 & 32 & 40 & 0.001050 & 142 & 143 & 40 & 0.004262 \\
\hline 32 & 33 & 40 & 0.001397 & 143 & 144 & 40 & 0.004609 \\
\hline 33 & 34 & 40 & 0.001744 & 144 & 145 & 40 & 0.004956 \\
\hline 34 & 35 & 40 & 0.002091 & 145 & 146 & 40 & 0.005303 \\
\hline 35 & 36 & 40 & 0.012091 & 146 & 147 & 40 & 0.005650 \\
\hline 36 & 37 & 40 & 0.012439 & 147 & 148 & 40 & 0.005998 \\
\hline 37 & 38 & 45 & 0.012830 & 148 & 149 & 40 & 0.006345 \\
\hline 38 & 64 & 45 & 0.013220 & 149 & 339 & 35 & 0.006648 \\
\hline 39 & 40 & 40 & 0.000355 & 157 & 158 & 40 & 0.000355 \\
\hline
\end{tabular}


J. Eng. Applied Sci., 14 (Special Issue 6): 9467-9476, 2019

Table 1: Continue

\begin{tabular}{|c|c|c|c|c|c|c|c|}
\hline \multicolumn{2}{|c|}{ Link from to } & \multirow{2}{*}{$\frac{\text { Length (m) }}{40}$} & \multirow{2}{*}{$\frac{\mathrm{Q} \text { design }\left(\mathrm{m}^{3} \mathrm{sec}^{-1}\right)}{0.000703}$} & \multicolumn{2}{|c|}{ Link from to } & \multirow{2}{*}{$\frac{\text { Length (m) }}{40}$} & \multirow{2}{*}{$\frac{Q \text { design }\left(\mathrm{m}^{3} \mathrm{sec}^{-1}\right)}{0.000703}$} \\
\hline 40 & 41 & & & 158 & 159 & & \\
\hline 41 & 42 & 40 & 0.001050 & 159 & 160 & 40 & 0.001050 \\
\hline 42 & 43 & 40 & 0.001397 & 156 & 160 & 20 & 0.000181 \\
\hline 43 & 44 & 40 & 0.001744 & 160 & 161 & 30 & 0.001483 \\
\hline 44 & 45 & 40 & 0.002091 & 161 & 165 & 30 & 0.001744 \\
\hline 45 & 56 & 40 & 0.002439 & 162 & 163 & 40 & 0.000355 \\
\hline 46 & 47 & 40 & 0.000355 & 163 & 164 & 40 & 0.000703 \\
\hline 47 & 48 & 35 & 0.000659 & 164 & 165 & 40 & 0.001050 \\
\hline 48 & 50 & 35 & 0.000963 & 165 & 166 & 30 & 0.003046 \\
\hline 49 & 50 & 40 & 0.000355 & 166 & 170 & 30 & 0.003306 \\
\hline 50 & 51 & 32 & 0.001588 & 167 & 168 & 40 & 0.000355 \\
\hline 51 & 55 & 30 & 0.001848 & 168 & 169 & 45 & 0.000746 \\
\hline 52 & 53 & 40 & 0.000355 & 169 & 170 & 45 & 0.001137 \\
\hline 53 & 54 & 35 & 0.000659 & 170 & 171 & 30 & 0.004695 \\
\hline 54 & 55 & 35 & 0.000963 & 171 & 175 & 30 & 0.004955 \\
\hline 55 & 56 & 50 & 0.003238 & 172 & 173 & 40 & 0.000355 \\
\hline 56 & 57 & 30 & 0.005928 & 173 & 174 & 45 & 0.000746 \\
\hline 57 & 62 & 30 & 0.006188 & 174 & 175 & 45 & 0.001137 \\
\hline 58 & 59 & 40 & 0.000355 & 175 & 176 & 30 & 0.006344 \\
\hline 59 & 60 & 40 & 0.000703 & 176 & 180 & 30 & 0.006605 \\
\hline 60 & 61 & 40 & 0.001050 & 177 & 178 & 40 & 0.000355 \\
\hline 61 & 62 & 40 & 0.001397 & 178 & 179 & 40 & 0.000703 \\
\hline 62 & 63 & 28 & 0.007820 & 179 & 180 & 45 & 0.001093 \\
\hline 63 & 64 & 30 & 0.008080 & 180 & 181 & 25 & 0.008124 \\
\hline 64 & 65 & 32 & 0.021570 & 181 & 182 & 30 & 0.008384 \\
\hline 65 & 70 & 35 & 0.021874 & 182 & 183 & 30 & 0.008645 \\
\hline 66 & 67 & 40 & 0.000355 & 183 & 188 & 30 & 0.008905 \\
\hline 67 & 68 & 45 & 0.000746 & 184 & 185 & 40 & 0.000355 \\
\hline 68 & 69 & 45 & 0.001137 & 185 & 186 & 40 & 0.000703 \\
\hline 69 & 70 & 45 & 0.001527 & 186 & 187 & 50 & 0.001137 \\
\hline 70 & 71 & 31 & 0.023662 & 187 & 188 & 50 & 0.001571 \\
\hline 72 & 73 & 40 & 0.000355 & 188 & 189 & 30 & 0.010728 \\
\hline 73 & 74 & 40 & 0.000703 & 189 & 193 & 30 & 0.010988 \\
\hline 74 & 76 & 40 & 0.001050 & 190 & 191 & 40 & 0.000355 \\
\hline 75 & 76 & 40 & 0.000355 & 191 & 192 & 40 & 0.000703 \\
\hline 76 & 77 & 32 & 0.003844 & 192 & 193 & 40 & 0.001050 \\
\hline 77 & 81 & 30 & 0.004105 & 193 & 194 & 30 & 0.012291 \\
\hline 78 & 79 & 40 & 0.000355 & 194 & 198 & 30 & 0.012551 \\
\hline 79 & 80 & 40 & 0.000703 & 195 & 196 & 40 & 0.000355 \\
\hline 80 & 81 & 40 & 0.001050 & 196 & 197 & 40 & 0.000703 \\
\hline 81 & $81 \mathrm{~A}$ & 29 & 0.005398 & 197 & 198 & 40 & 0.001050 \\
\hline 150 & 151 & 40 & 0.000355 & 198 & 209 & 56 & 0.014080 \\
\hline 151 & 152 & 40 & 0.000703 & 199 & 200 & 40 & 0.000355 \\
\hline 152 & 154 & 40 & 0.001050 & 200 & 201 & 45 & 0.000746 \\
\hline 153 & 154 & 30 & 0.000268 & 201 & 202 & 45 & 0.001137 \\
\hline 154 & 155 & 40 & 0.001657 & 202 & 206 & 55 & 0.001615 \\
\hline 155 & $81 \mathrm{~A}$ & 20 & 0.001830 & 203 & 204 & 40 & 0.000355 \\
\hline $81 \mathrm{~A}$ & 82 & 12 & 0.007324 & 204 & 205 & 45 & 0.000746 \\
\hline 82 & 83 & 40 & 0.007673 & 205 & 206 & 45 & 0.001137 \\
\hline 83 & 84 & 40 & 0.008020 & 206 & 207 & 40 & 0.003090 \\
\hline 84 & 85 & 45 & 0.008411 & 207 & 208 & 45 & 0.003828 \\
\hline 85 & 86 & 45 & 0.008802 & 208 & 209 & 45 & 0.004956 \\
\hline 86 & 87 & 45 & 0.009192 & 209 & 210 & 20 & 0.019200 \\
\hline 87 & 88 & 30 & 0.009452 & 210 & 211 & 38 & 0.019531 \\
\hline 88 & 92 & 30 & 0.009712 & 211 & 212 & 45 & 0.019922 \\
\hline 89 & 90 & 40 & 0.000355 & 212 & 213 & 45 & 0.020312 \\
\hline 90 & 91 & 45 & 0.000746 & 213 & 338 & 45 & 0.020703 \\
\hline 91 & 92 & 45 & 0.001137 & 338 & 339 & 25 & 0.020919 \\
\hline 92 & 93 & 30 & 0.011101 & 339 & 340 & 30 & 0.027820 \\
\hline 93 & 97 & 30 & 0.011362 & 340 & 341 & 29 & 0.028072 \\
\hline 94 & 95 & 40 & 0.000355 & 341 & 342 & 30 & 0.029634 \\
\hline 95 & 96 & 45 & 0.000746 & 342 & 343 & 30 & 0.029895 \\
\hline 96 & 97 & 45 & 0.001137 & 343 & 344 & 30 & 0.031457 \\
\hline 97 & 98 & 30 & 0.012751 & 344 & 345 & 30 & 0.031718 \\
\hline 98 & 102 & 30 & 0.013011 & 345 & 346 & 30 & 0.035450 \\
\hline 99 & 100 & 40 & 0.000355 & 346 & 347 & 30 & 0.035711 \\
\hline 100 & 101 & 45 & 0.000746 & 347 & 348 & 30 & 0.038098 \\
\hline 101 & 102 & 45 & 0.001137 & 348 & 349 & 30 & 0.055320 \\
\hline 102 & 103 & 30 & 0.014400 & & 8227 & & \\
\hline
\end{tabular}


J. Eng. Applied Sci., 14 (Special Issue 6): 9467-9476, 2019

Table 2: Optimum layout design for the case study by proposed model

\begin{tabular}{|c|c|c|c|c|c|c|c|}
\hline \multicolumn{2}{|c|}{ Pipe from to } & \multirow{2}{*}{$\frac{\text { Length (m) }}{40}$} & \multirow{2}{*}{$\frac{\mathrm{Q} \text { design }\left(\mathrm{m}^{3} \mathrm{sec}^{-1}\right)}{0.000355}$} & \multicolumn{2}{|c|}{ Pipe from to } & \multirow{2}{*}{$\begin{array}{l}\text { Length (m) } \\
45\end{array}$} & \multirow{2}{*}{$\begin{array}{c}\mathrm{Q} \text { design }\left(\mathrm{m}^{3} \mathrm{sec}^{-1}\right) \\
0.000746\end{array}$} \\
\hline$\overline{1}$ & 2 & & & 100 & 101 & & \\
\hline 2 & 3 & 50 & 0.000790 & 101 & 102 & 45 & 0.001137 \\
\hline 3 & 4 & 50 & 0.001224 & 102 & 103 & 30 & 0.014400 \\
\hline 4 & 5 & 29 & 0.001474 & 103 & 107 & 30 & 0.014660 \\
\hline 5 & 10 & 30 & 0.001735 & 104 & 105 & 40 & 0.000355 \\
\hline 6 & 7 & 40 & 0.000355 & 105 & 106 & 45 & 0.000746 \\
\hline 7 & 8 & 45 & 0.000746 & 106 & 107 & 45 & 0.001137 \\
\hline 8 & 9 & 45 & 0.001137 & 107 & 110 & 56 & 0.016276 \\
\hline 9 & 10 & 45 & 0.001527 & 108 & 109 & 40 & 0.000355 \\
\hline 10 & 11 & 29 & 0.003506 & 109 & 110 & 40 & 0.000703 \\
\hline 11 & 16 & 30 & 0.003766 & 110 & 348 & 30 & 0.017230 \\
\hline 12 & 13 & 40 & 0.000355 & 112 & 113 & 50 & 0.000443 \\
\hline 13 & 14 & 45 & 0.000746 & 113 & 115 & 50 & 0.000877 \\
\hline 14 & 15 & 45 & 0.001137 & 115 & 116 & 50 & 0.025299 \\
\hline 15 & 16 & 45 & 0.001527 & 116 & 347 & 50 & 0.025734 \\
\hline 16 & 17 & 28 & 0.005528 & 119 & 120 & 40 & 0.000355 \\
\hline 17 & 22 & 30 & 0.005789 & 120 & 121 & 40 & 0.000703 \\
\hline 18 & 19 & 40 & 0.000355 & 121 & 122 & 35 & 0.001006 \\
\hline 19 & 20 & 45 & 0.000746 & 122 & 345 & 35 & 0.001310 \\
\hline 20 & 21 & 45 & 0.001137 & 125 & 126 & 40 & 0.000355 \\
\hline 21 & 22 & 45 & 0.001527 & 126 & 127 & 40 & 0.000703 \\
\hline 22 & 23 & 33 & 0.007595 & 127 & 128 & 35 & 0.001006 \\
\hline 23 & 28 & 30 & 0.007855 & 128 & 343 & 35 & 0.001310 \\
\hline 24 & 25 & 40 & 0.000355 & 131 & 132 & 40 & 0.000355 \\
\hline 25 & 26 & 45 & 0.000746 & 132 & 133 & 40 & 0.000703 \\
\hline 26 & 27 & 45 & 0.001137 & 133 & 134 & 35 & 0.001006 \\
\hline 27 & 28 & 45 & 0.001527 & 134 & 341 & 35 & 0.001310 \\
\hline 28 & 35 & 33 & 0.009660 & 137 & 138 & 40 & 0.000355 \\
\hline 29 & 30 & 40 & 0.000355 & 138 & 139 & 40 & 0.002873 \\
\hline 30 & 31 & 40 & 0.000703 & 139 & 140 & 40 & 0.003220 \\
\hline 31 & 32 & 40 & 0.001050 & 145 & 144 & 45 & 0.000399 \\
\hline 32 & 33 & 40 & 0.001397 & 144 & 143 & 40 & 0.000746 \\
\hline 33 & 34 & 40 & 0.001744 & 143 & 142 & 40 & 0.001093 \\
\hline 34 & 35 & 40 & 0.002091 & 142 & 141 & 40 & 0.001440 \\
\hline 35 & 36 & 40 & 0.012091 & 141 & 140 & 40 & 0.001788 \\
\hline 36 & 37 & 40 & 0.012439 & 140 & 115 & 55 & 0.023998 \\
\hline 37 & 38 & 45 & 0.012830 & 146 & 147 & 35 & 0.000312 \\
\hline 38 & 64 & 45 & 0.013220 & 147 & 148 & 40 & 0.000659 \\
\hline 39 & 40 & 40 & 0.000355 & 148 & 149 & 40 & 0.001006 \\
\hline 40 & 41 & 40 & 0.000703 & 149 & 339 & 35 & 0.001310 \\
\hline 41 & 42 & 40 & 0.001050 & 157 & 158 & 40 & 0.000355 \\
\hline 42 & 43 & 40 & 0.001397 & 158 & 159 & 40 & 0.000703 \\
\hline 43 & 44 & 40 & 0.001744 & 159 & 160 & 40 & 0.001050 \\
\hline 44 & 45 & 40 & 0.002091 & 156 & 160 & 20 & 0.000181 \\
\hline 45 & 56 & 40 & 0.002439 & 160 & 161 & 30 & 0.001483 \\
\hline 46 & 47 & 40 & 0.000355 & 161 & 165 & 30 & 0.001744 \\
\hline 47 & 48 & 35 & 0.000659 & 162 & 163 & 40 & 0.000355 \\
\hline 48 & 50 & 35 & 0.000963 & 163 & 164 & 40 & 0.000703 \\
\hline 49 & 50 & 40 & 0.000355 & 164 & 165 & 40 & 0.001050 \\
\hline 50 & 51 & 32 & 0.001588 & 165 & 166 & 30 & 0.003046 \\
\hline 51 & 55 & 30 & 0.001848 & 166 & 170 & 30 & 0.003306 \\
\hline 52 & 53 & 40 & 0.000355 & 167 & 168 & 40 & 0.000355 \\
\hline 53 & 54 & 35 & 0.000659 & 168 & 169 & 45 & 0.000746 \\
\hline 54 & 55 & 35 & 0.000963 & 169 & 170 & 45 & 0.001137 \\
\hline 55 & 56 & 50 & 0.003238 & 170 & 171 & 30 & 0.004695 \\
\hline 56 & 57 & 30 & 0.005928 & 171 & 175 & 30 & 0.004955 \\
\hline 57 & 62 & 30 & 0.006188 & 172 & 173 & 40 & 0.000355 \\
\hline 58 & 59 & 40 & 0.000355 & 173 & 174 & 45 & 0.000746 \\
\hline 59 & 60 & 40 & 0.000703 & 174 & 175 & 45 & 0.001137 \\
\hline 60 & 61 & 40 & 0.001050 & 175 & 176 & 30 & 0.006344 \\
\hline 61 & 62 & 40 & 0.001397 & 176 & 180 & 30 & 0.006605 \\
\hline 62 & 63 & 28 & 0.007820 & 177 & 178 & 40 & 0.000355 \\
\hline 63 & 64 & 30 & 0.008080 & 178 & 179 & 40 & 0.000703 \\
\hline 64 & 65 & 32 & 0.021570 & 179 & 180 & 45 & 0.001093 \\
\hline 65 & 70 & 35 & 0.021874 & 180 & 181 & 25 & 0.008124 \\
\hline 66 & 67 & 40 & 0.000355 & 181 & 182 & 30 & 0.008384 \\
\hline 67 & 68 & 45 & 0.000746 & 183 & 182 & 30 & 0.009886 \\
\hline
\end{tabular}


J. Eng. Applied Sci., 14 (Special Issue 6): 9467-9476, 2019

\begin{tabular}{|c|c|c|c|c|c|c|c|}
\hline \multicolumn{2}{|c|}{ Pipe from to } & \multirow{2}{*}{$\frac{\text { Length (m) }}{45}$} & \multirow{2}{*}{$\frac{\mathrm{Q} \text { design }\left(\mathrm{m}^{3} \mathrm{sec}^{-1}\right)}{0.001137}$} & \multicolumn{2}{|c|}{ Pipe from to } & \multirow{2}{*}{$\frac{\text { Length }(\mathrm{m})}{30}$} & \multirow{2}{*}{$\frac{\mathrm{Q} \text { design }\left(\mathrm{m}^{3} \mathrm{sec}^{-1}\right)}{0.009626}$} \\
\hline$\overline{68}$ & 69 & & & 188 & 183 & & \\
\hline 69 & 70 & 45 & 0.001527 & 184 & 185 & 50 & 0.001970 \\
\hline 70 & 71 & 31 & 0.023662 & 185 & 186 & 40 & 0.003845 \\
\hline 72 & 73 & 40 & 0.000355 & 186 & 187 & 50 & 0.004280 \\
\hline 73 & 74 & 40 & 0.000703 & 187 & 188 & 50 & 0.004714 \\
\hline 74 & 76 & 40 & 0.001050 & 189 & 188 & 30 & 0.004660 \\
\hline 75 & 76 & 40 & 0.000355 & 193 & 189 & 30 & 0.004400 \\
\hline 76 & 77 & 32 & 0.003844 & 190 & 191 & 40 & 0.000355 \\
\hline 77 & 81 & 30 & 0.004105 & 191 & 192 & 40 & 0.000703 \\
\hline 78 & 79 & 40 & 0.000355 & 192 & 193 & 40 & 0.001050 \\
\hline 79 & 80 & 40 & 0.000703 & 194 & 193 & 30 & 0.003098 \\
\hline 80 & 81 & 40 & 0.001050 & 198 & 194 & 30 & 0.002837 \\
\hline 81 & $81 \mathrm{~A}$ & 29 & 0.005398 & 195 & 196 & 40 & 0.000355 \\
\hline 150 & 151 & 40 & 0.000355 & 196 & 197 & 40 & 0.000703 \\
\hline 151 & 152 & 40 & 0.000703 & 197 & 198 & 40 & 0.001050 \\
\hline 152 & 154 & 40 & 0.001050 & 209 & 198 & 56 & 0.001537 \\
\hline 153 & 154 & 30 & 0.000268 & 182 & 140 & 30.5 & 0.018528 \\
\hline 154 & 155 & 40 & 0.001657 & 202 & 201 & 45 & 0.000399 \\
\hline 155 & $81 \mathrm{~A}$ & 20 & 0.001830 & 201 & 200 & 45 & 0.000790 \\
\hline $81 \mathrm{~A}$ & 82 & 12 & 0.007324 & 200 & 199 & 40 & 0.001137 \\
\hline 82 & 83 & 40 & 0.007673 & 199 & 184 & 46 & 0.001536 \\
\hline 83 & 84 & 40 & 0.008020 & 206 & 205 & 45 & 0.000399 \\
\hline 84 & 85 & 45 & 0.008411 & 205 & 204 & 45 & 0.000790 \\
\hline 85 & 86 & 45 & 0.008802 & 204 & 203 & 40 & 0.001137 \\
\hline 86 & 87 & 45 & 0.009192 & 203 & 185 & 46 & 0.001536 \\
\hline 87 & 88 & 30 & 0.009452 & $206 \mathrm{~A}$ & 207 & 30 & 0.000268 \\
\hline 88 & 92 & 30 & 0.009712 & 207 & 208 & 45 & 0.000659 \\
\hline 89 & 90 & 40 & 0.000355 & 208 & 209 & 45 & 0.001050 \\
\hline 90 & 91 & 45 & 0.000746 & 339 & 340 & 30 & 0.001570 \\
\hline 91 & 92 & 45 & 0.001137 & 340 & 341 & 29 & 0.001822 \\
\hline 92 & 93 & 30 & 0.011101 & 341 & 342 & 30 & 0.003384 \\
\hline 93 & 97 & 30 & 0.011362 & 342 & 343 & 30 & 0.003645 \\
\hline 94 & 95 & 40 & 0.000355 & 343 & 344 & 30 & 0.005207 \\
\hline 95 & 96 & 45 & 0.000746 & 344 & 345 & 30 & 0.005468 \\
\hline 96 & 97 & 45 & 0.001137 & 345 & 346 & 30 & 0.009200 \\
\hline 97 & 98 & 30 & 0.012751 & 346 & 347 & 30 & 0.009461 \\
\hline 98 & 102 & 30 & 0.013011 & 347 & 348 & 30 & 0.035446 \\
\hline 99 & 100 & 40 & 0.000355 & 348 & 349 & 30 & 0.052668 \\
\hline
\end{tabular}

\section{CONCLUSION}

The adaptive Genetic Algorithm procedure have been successfully developed-in a hybrid optimization model which combines GAs with a TGA to enable optimal tree networks to be selected from directed base graphs of sewer network. The drawbacks of using this approach are few and the bene ts are such that optimization runs can either be made shorter to achieve a given goal or discover better results in a xed timeframe. These algorithms require only limited computer facilities and can be used to design the layout of large nonlinear flow networks.

Comparison of the solution for the benchmark example obtained by proposed model with reduction in search space, with the solutions provided by Walters and Smith, 1995, indicated that the modified model is more efficient and effective in finding similar result (5218 u nits) with much reduced computational effort. As well as the lack of the need to repair or discard infeasible layouts or even apply the factors of penalty on the cost function. This improves the performance of the optimization model more efficiently in terms of speed and accuracy.
In order to ensure the efficiency of the proposed method for the design of real networks, it was examined with case study located in Karbala Holy city, Iraq, then compared the cost of the manual designs with the designs obtained from this model for networks. The saving percentage was $13.05 \%$, the savings percentage obtained through the optimal design by using the proposed GA-TGA model indicate that the model is well performing.

\section{ACKNOWLEDGMENT}

The authors would like to thank the faculty members of Kerbala University/College of Engineering and all members of the civil engineering department for their help.

\section{REFERENCES}

Baker, J.E., 1987. Reducing bias and inefficiency in the selection algorithm. Proceedings of the 2nd International Conference on Genetic Algorithms on Genetic Algorithms and their Application, July 1987, Cambridge, Massachusetts, United States, pp: 14-21. 
Davidson, J.W. and I.C. Goulter, 1995. Evolution program for design of rectilinear branched networks. J. Comput. Civil Eng., 9: 112-121.

Davidson, J.W., 1999. Evolution program for layout geometry of rectilinear looped networks. J. Comput. Civil Eng., 13: 246-253.

De Jong, K.A., 1975. An analysis of the behavior of a class of genetic adaptive systems. Ph.D. Thesis, Department of Computer and Communication Sciences, University of Michigan, Ann Arbor.

Geem, Z.W., J.H. Kim and Y.N. Yoon, 2000. Optimal layout of pipe networks using harmony search. Proceedings of 4th International Conference on Hydro-Science and Engineering, September 26-29, 2000, Korea Water Resources Association South Korea, pp: 199-212.

Goldberg, D.E. and S. Voessner, 1999. Optimizing global-local search hybrids. Proceedings of the 1st Annual Conference on Genetic and Evolutionary Computation Volume 1, July 13-17, 1999, Morgan Kaufmann Publishers Inc., Massachusetts, USA., pp: 220-228.

Goldberg, D.E., 1989. Genetic Algorithms in Search Optimization and Machine Learning. Addison-Wesley, New York, USA.

Grefenstette, J.J., R. Gopal, B. Rosmaita and D. Van Gucht, 1985. Genetic algorithms for the traveling salesman problem. Proceedings of the 1st International Conference on Genetic Algorithms and their Application, July 24-26, 1985, Boston, pp: 160-168.

Hassan, W.H. and Z.H. Atiyah, 2019. Optimum layout design of sewer networks by hybrid genetic algorithm. J. Applied Water Eng. Res., (In Press).

Hassan, W.H., 2019. Application of a genetic algorithm for the optimization of a location and inclination angle of a cut-off wall for anisotropic foundations under hydraulic structures. Geotechn. Geol. Eng., 37: 883-895.

Hassan, W.H., M.H. Jassem and S.S. Mohammed, 2018. A GA-HP model for the optimal design of sewer networks. Water Resour. Manage., 32: 865-879.
Holland, J.H., 1975. Adaptation in Natural and Artificial Systems: An Introductory Analysis with Applications to Biology, Control and Artificial Intelligence. 1st Edn., University of Michigan Press, Ann Arbor, MI., USA., ISBN-13: 9780472084609, Pages: 183.

Jebari, K. and M. Madiafi, 2013. Selection methods for genetic algorithms. Intl. J. Emerg. Sci., 3: 333-344.

Mays, L.W. and H.G. Wenzel Jr., 1976. Optimal design of multilevel branching sewer systems. Water Resour. Res., 12: 913-917.

Mays, L.W., J.C. Liebman and H.G. Wenzel Jr., 1976. Model for layout and design of sewer systems. J. Water Resour. Plann. Manage. Div., 102: 385-405.

Miettinen, K., P. Neittaanmaki and M.M. Makela, 1999. Evolutionary Algorithms in Engineering and Computer Science. Wiley, New York.

Morgan, D.R. and I.C. Goulter, 1985. Optimal urban water distribution design. Water Resour. Res., 21: 642-652.

Rowell, W.F. and J. Barnes, 1982. Obtaining the layout of water distribution systems. J. Hydraulics Div., 108: 137-148.

Smith, D., 1985. Bin-packing with adaptive search. Proceedings of an International Conference on Genetic Algorithms and their Applications, July 24-26, 1985, Lawrence Erlbaum, pp: 202-206.

Walski, T.M., 1985. State-of-the-art pipe network optimization. Proceedings of the International Conferences on Computer Applications in Water Resources, June 10-12, 1985, American Society of Civil Engineers, New York, USA., pp: 559-568.

Walters, G.A. and D.K. Smith, 1995. Evolutionary design algorithm for optimal layout of tree networks. Eng. Optim., 24: 261-281.

Walters, G.A. and T. Lohbeck, 1993. Optimal layout of tree networks using genetic algorithms. Eng. Optim., 22: 27-48.

Walters, G.A., 1992. A Review of Pipe Network Optimization Techniques. In: Pipeline Systems, Coulbeck, B. and E.P. Evans (Eds.). Springer, Dordrecht, Netherlands, ISBN: 978-90-481-4143-2, pp: 3-13. 